\title{
Ramsauer-Townsend minimum in methane - modified effective range analysis
}

\author{
Kamil Fedus and Grzegorz P. Karwasz ${ }^{\mathrm{a}}$ \\ Institute of Physics, Faculty of Physics, Astronomy and Informatics, Nicolaus Copernicus University, Grudziadzka 5, \\ 87-100 Toruń, Poland
}

Received 22 November 2013/ Received in final form 29 January 2014

Published online 24 April 2014

(c) The Author(s) 2014. This article is published with open access at Springerlink.com

\begin{abstract}
Electron-scattering cross sections in methane are analysed in the very-low energy region. The correspondence between integral elastic, elastic differential and momentum transfer cross sections is checked via a novel approach to modified effective range theory, in order to determine the depth and position of the Ramsauer-Townsend minimum. Phase shifts of the two lowest partial waves are obtained explicitly and parameterized by four coefficients with the physical meaning of the scattering lengths and the effective ranges. Using recent experiments on vibrational cross sections performed over an extended $\left(0-180^{\circ}\right)$ angular range and comparing several theories, an agreement within $10 \%$ has been obtained between experimental total and present summed (elastic + vibrational) cross sections in the whole 0.1-2.0 eV energy range. An additional check for consistency is done using two-term Boltzmann analysis to derive electron diffusion coefficients. Calculated drift velocities and transversal diffusion coefficients at $0-10 \mathrm{Td}$ reduced electric field agree within $5 \%$ with experiments.
\end{abstract}

\section{Introduction}

The discovery of the Ramsauer-Townsend (R-T) minimum, observed for electron scattering in $\mathrm{Ar}$ at about $0.3 \mathrm{eV}$, independently in swarm and beam electron scattering experiments $[1,2]$, triggered the development of quantum wave theories. The R-T minimum occurs in heavier atomic gases, where the scattering potential is strong enough to induce a $\pi(\operatorname{modulo} \pi)$ phase-shift in the $s$-partial wave at low impact energies, where contributions from other partial waves are still small. It was shown previously in pioneering work for argon [3] that including an empirical polarization potential is necessary to reproduce theoretically the R-T minimum. The sub-eV electron energies are important for tokomak edge regions, radiation counters and electrical discharges [4,5], with the R-T minimum determining electron temperatures in plasmas [6].

In molecules, a low-energy minimum was previously observed experimentally in the total cross section in $\mathrm{CH}_{4}$ by Ramsauer and Kollath [7]. R-T is also present in $\mathrm{SiH}_{4}$ and $\mathrm{GeH}_{4}$, see $[8,9]$ for comparison. In $\mathrm{N}_{2}$, Ramanan and Freeman [10] in their low-temperature swarm measurements "did not exclude the presence of R-T minimum" at energies as low as a few meV, but no clear experimental or theoretical evidence is available from other studies. In another tetrahedral molecule, $\mathrm{CF}_{4}$, the $\mathrm{R}$ - $\mathrm{T}$ in the elastic cross section, possibly $0.6 \AA$ at $0.1 \mathrm{eV}$, see [9], is masked in the total cross section by strong vibrational ex-

\footnotetext{
a e-mail: karwasz@fizyka.umk.pl
}

citation. Shallow "valleys" in total cross sections are visible in $\mathrm{H}_{2} \mathrm{O}$ and $\mathrm{NH}_{3}$ [11], but the possible presence of an $\mathrm{R}-\mathrm{T}$ minimum is masked by strong rotational excitation, see analysis in [8]. Excluding rotations from experimental vibrationally elastic cross sections [12] would yield an R-T minimum in elastic cross section in $\mathrm{H}_{2} \mathrm{O}$ of about $2 \AA^{2}$ at $2 \mathrm{eV}$ but both the experiment and the analysis are tedious and can lead to serious errors, see [8].

The methane molecule remains, therefore, the lightest target for which an R-T minimum is clearly seen. This is surely due to its high polarizability, estimated from experiments and theory to be between $\alpha=16-21 a_{0}^{3}[13,14]$, as compared to isoelectronic neon $\left(2.63 \mathrm{a}_{0}^{3}\right)$ or argon $\left(11.1 a_{0}^{3}\right)$. However, also in $\mathrm{CH}_{4}$, the depth of the $\mathrm{R}-\mathrm{T}$ minimum is masked in the experimental total cross section by a maximum of the vibrational excitation, particularly of the two overlapping $\left(v_{2}\right.$ and $\left.v_{4}\right)$ deformation modes. The high value of the polarizability makes scattering forward centred [15], even at low energies, requiring measurements at small angles in order to evaluate properly the integral cross sections.

For the theoretical approach, methane is one of the most intensively studied molecules [16-28]. The high value of dipole polarizability makes it difficult to determine theoretically the low energy elastic collisions parameters. Different asymptotic models of the polarization potential influence significantly the depth and the position of the R-T minimum, so alternative forms of polarization are frequently used in separate energy regions $[16,17,24,25]$. Therefore, no consensus has been obtained on $\mathrm{CH}_{4}$ cross 
sections in the R-T minimum range so far, as it has also been documented by numerous alternative sets of data used for modelling electron diffusion processes and gas discharges in methane and its mixtures [27,29].

In the present work we re-analyse available experimental data on differential, integral and momentum transfer elastic cross sections for electron scattering by methane in the region of the $\mathrm{R}-\mathrm{T}$ minimum. In particular we used recent elastic (and vibrational) cross sections obtained in a wide angular range with the use of the magnetic angle-changer by Allan [30,31]. A mutual correspondence between complementary data was obtained via a novel [32-34] approach to the modified effective range theory.

The new form of MERT has recently been tested in the energy range up to a few $\mathrm{eV}$ for electron and positron scattering by $\mathrm{He}, \mathrm{Ar}, \mathrm{H}_{2}$ and (in a preliminary way) $\mathrm{CH}_{4}$ [34]. As input data, experimental total cross sections were used; in noble gases they coincide with integral elastic cross sections in the considered energy range. The model [34] worked well for $\mathrm{He}, \mathrm{Ar}$ and $\mathrm{H}_{2}$, but in $\mathrm{CH}_{4}$ the contribution from the vibrational channel to the total cross sections has to be considered even at low energies.

Therefore, in the present analysis, the elastic cross sections in $\mathrm{CH}_{4}$ are completed with experimental and theoretical data on vibrational excitation and compared with absolute measurements of total cross sections [35-37]. The Boltzmann two-term model [38] is used additionally to check the consistency of elastic and vibrational cross sections with experimental electron-drift coefficients. Consequently, a self-consistent set of cross sections in the region of the $\mathrm{R}$ - $\mathrm{T}$ minimum in $\mathrm{CH}_{4}$ is proposed.

\section{Modified effective range approach}

Modified effective range theory (MERT) was developed $[39,40]$ in order to extend the concept of the effective range to the polarization interaction $\left(\sim r^{-4}\right)$ dominating low-energy electron and positron-atom scattering [39]. Standard applications of MERT to, for example, noble gases [41] are based on experimental elastic integral cross sections and use four (or six) parameters expanding the two lowest partial-wave phase shifts $\eta_{l}$ into a series of wavenumber $k$ powers, as follows:

$$
\begin{aligned}
\tan \eta_{0}(k)= & -A k-4 \alpha A k^{3} \ln \left(k a_{0}\right) / 3 a_{0} \\
& -\pi \alpha k^{2} / 3 a_{0}+D k^{3}+F k^{4} \\
\tan \eta_{1}(k)= & \pi \alpha k^{2} / 15 a_{0}-A_{1} k^{3}
\end{aligned}
$$

where $l=0,1$ is the angular momentum quantum number, $\alpha$ is the target dipole polarizability and $a_{0}$ is the atomic unit of length (Bohr radius). Out of the parameters used, only $A$ has a physical meaning, namely of the scattering length, giving the zero energy integral elastic $\sigma_{I E}$ and momentum transfer $\sigma_{M T}$ cross sections $\sigma_{I E}(E=0)=\sigma_{M T}(E=0)=4 \pi A^{2}$. The applicability of MERT in the original form was limited to a very low energy regime, no higher than $1 \mathrm{eV}$, as was extensively discussed by Buckman and Mitroy [41] for noble gases.
In methane, several works used the above expansion of $\sigma_{I E}$ into a series of the wave number $k$ in the zeroenergy limit. Ferch et al. [35] used four parameters to model their total cross sections below $0.5 \mathrm{eV}$ of impact energy; Schmidt [29] in his analysis including the $d$-wave shift used six parameters (see Ref. [29] for detailed explanations). Four-parameter MERT was used also by Lunt et al. $[42,43]$ to analyse backward-scattering cross sections in the $0.013-0.175 \mathrm{eV}$ energy range.

The main limitation of expansions like equations (1) and (2) is the divergence of alternating signs of higherorder terms in the power series. In order to extend the energy range of validity, Idziaszek and Karwasz proposed [32] an alternative approach to MERT. In that method the contribution of the long-range particle-target interaction to the scattering phase-shifts is solved exactly using the properties of Mathieu functions - the analytical solutions of the Schrödinger equation with polarization potential while the effective range approximation is introduced exclusively for the short-range interaction. In this way, the fitting parameters of the effective range expansions given by equations (1) and (2) are replaced by two coefficients characterizing the scattering potential, with the physical meaning of the scattering length and the effective range. In this paper we show that for methane only the two lowest partial waves are sufficient for the explicit analysis of electron-methane collisional data below $2 \mathrm{eV}$ of impact energy.

In detail, the model [32] describes the phase shifts $\eta_{l}$ as follows:

$$
\tan \eta_{l}=\frac{m^{2}-\tan ^{2} \delta_{l}+B_{l} \tan \delta_{l}\left(m^{2}-1\right)}{\tan \delta_{l}\left(1-m^{2}\right)+B_{l}\left(1-m^{2} \tan ^{2} \delta_{l}\right)}
$$

where $\delta_{l}=\pi(\nu-l-1 / 2) / 2$. Here $m$ and $\nu$ denote the energy-dependent parameters that have to be determined numerically from the analytical properties of Mathieu functions, see [32]. The contribution of the short-range interaction is hidden in the parameter $B_{l}(k)$ related to the additional phase shift that is induced by the shortrange potential. This parameter is expanded around zero energy for each partial wave separately: $B_{l}(k) \approx B_{l}(0)+$ $R_{l} R^{*} k^{2} / 2+\ldots$, where $R_{l}$ can be interpreted as the effective range for a given partial wave. Here $R^{*}=\sqrt{\alpha e^{2} \mu / h^{2}}$ denotes a typical length scale related to the $r^{-4}$ interaction, with $e$ being the elementary charge, $\mu$ the reduced mass of projectile/target system and $\hbar$ the Planck constant. In the particular case of $l=0, B_{0}(0)$ can be expressed in terms of $A$, the $s$-wave scattering length, as $B_{0}=-R^{*} / A$.

The number of partial waves necessary to be treated by equation (4) can be estimated by comparing the energy of the projectile with the height of the centrifugal barrier: $E_{b}(l)=1 / 4 E^{*} l^{2}(l+1)^{2}$ for the $r^{-4}$ interaction [44], where $E^{*}=h^{2} / 2 \mu R^{* 2}$ is the characteristic energy. For methane the characteristic energy, i.e. the range of the expected validity of MERT, is $E^{*}=0.72 \mathrm{eV}$ while the characteristic range is $R^{*}=4.36 a_{0}$. The $l$ th partial wave of the projectile can probe deeply the region of the short-range interaction only when the particle energy $E>E_{b}(l)$. When the 
Table 1. MERT parameters for electron scattering in methane (some previous results $[29,36]$ ), see text for explanations.

\begin{tabular}{ccccccc}
\hline MERT parameters & $A\left(a_{0}\right)$ & $D\left(a_{0}^{3}\right)$ & $F\left(a_{0}^{4}\right)$ & $A_{1}\left(a_{0}^{3}\right)$ & $H\left(a_{0}^{5}\right)$ & $A_{2}\left(a_{0}^{5}\right)$ \\
\hline Ferch et al. [35] & -2.475 & 186.0 & -337 & 10.1 & & \\
Schmidt [29] & -2.755 & 171.2 & -92.2 & 17.01 & 208.05 & 66.63 \\
\hline
\end{tabular}

particle cannot overcome the centrifugal barrier at a given angular momentum, the scattering phase-shift can be approximated by the following relation [39]:

$$
\tan \eta_{l}(k) \approx \frac{\pi \alpha k^{2}}{8(l-1 / 2)(l+1 / 2)(l+3 / 2)}, \text { for } l>1
$$

Integral elastic $\left(\sigma_{I E}\right)$, momentum transfer $\left(\sigma_{M T}\right)$ and differential elastic $(d \sigma / d \omega)$ cross sections are calculated using the following relations:

$$
\begin{aligned}
\sigma_{I E} & =\frac{4 \pi}{k^{2}} \sum_{l=0}^{\infty}(2 l+1) \sin ^{2} \eta_{l}(k) \\
\sigma_{M T} & =\frac{4 \pi}{k^{2}} \sum_{l=0}^{\infty}(l+1) \sin ^{2}\left[\eta_{l}(k)-\eta_{l+1}(k)\right] \\
\frac{d \sigma}{d \omega} & =\frac{1}{k^{2}}\left|\sum_{l=0}^{\infty}(2 l+1) \exp \left(i \eta_{l}\right) \sin \left(\eta_{l}\right) P_{l}(\cos \theta)\right|^{2}
\end{aligned}
$$

where $P_{l}(x)$ are the Legendre polynomials and $\theta$ is the scattering angle.

Substituting equation (3) into equations (5)-(7) one gets relations which can be fitted to experimental data in order to determine the unknown parameters of the effective range expansion of $B_{l}(k)$. As shown below, only two partial waves $(s$ and $p$ ) are sufficient to describe the cross-sections for electron collisions with $\mathrm{CH}_{4}$ up to an impact energy of $2 \mathrm{eV}$; hence only four fitting parameters are used in the current analysis. The physical interpretation of these parameters can be described as follows: $A$ is the $s$-wave scattering length (related to $B_{0}$ ), $B_{1}$ is the zeroenergy contribution of $p$-wave, and $R_{0}$ and $R_{1}$ represents the $s$-wave and the $p$-wave effective ranges, respectively. In all our calculations we will consider also the contribution of 100 higher partial waves with $l>1$ using the Born phase-shift approximation, equation (4).

Through extensive comparative analysis we found that the best results are obtained when using a value of dipole polarizability $\alpha \approx 19 a_{0}^{3}$ that is slightly higher than the experimental value $\alpha \approx 17.5 a_{0}^{3}$ [14] and the values 18.0 $18.2 a_{0}^{3}$ calculated with polarized virtual orbitals [21]. The value used presently proved to fit better the experimental data chosen as described below. The difference in $\alpha$ can reflect the octupole moment of $\mathrm{CH}_{4}$ (see later) or some short-range interactions, not included in present analysis.

Interactive cross-check procedures using several experimental low-energy cross section sets were used. The data included: (1) elastic differential and elastic integral cross sections (Sohn et al. [18]); (2) elastic differential and vibrational differential of Allan [31] - presently integrated numerically to derive integral elastic and vibrational cross sections - and one of the representative theories [45];
Table 2. MERT parameters for electron scattering in methane (present analysis): $A=-R^{*} / B_{0}$ is the scattering length, $B_{1}$ is the zero energy contribution of the $p$-wave, $R_{0}$ - the $s$-wave effective range and $R_{1}$ - the $p$-wave effective range. Note a different meaning of present parameters, apart from $A$, than those in Table 1.

\begin{tabular}{cccc}
\hline \multicolumn{4}{c}{ Present MERT parameters } \\
\hline$A\left(a_{0}\right)$ & $R_{0}(a)$ & $B_{1}$ & $R_{1}(a)$ \\
-2.80 & -0.63 & -0.60 & -0.27 \\
\hline
\end{tabular}

(3) swarm-derived [29] momentum transfer cross sections and (4) grand total cross sections [35-37]. As a starting point for comparisons we used elastic differential cross sections (DCS) at selected energies [17] and swarm-derived momentum transfer cross sections [29], but those data alone did not allow us to obtain a mutual consistency. A decisive breakthrough in the analysis was obtained using the most recent absolute elastic DCS at $45^{\circ}, 90^{\circ}, 135^{\circ}$ and $180^{\circ}$, measured as a continuous function of energy in the whole range from 0.2 to $2 \mathrm{eV}$ [31]. This allowed earlier beam and swarm data also to be brought to a mutual agreement.

\section{Present MERT results}

Our recent analysis in $\mathrm{CH}_{4}$ [34] was limited to total cross sections. We are aware that only differential cross section (DCS) at several energies (or energy dependences of DCS at fixed angles) constitutes a truly stringent test for the theory. In the present analysis we consider an extensive set of experimental data and in particular we include the most recent DCS obtained with the magnetic-field angle analyser by Allan [31]. This new technique allows us to extend the range of differential CS measurements from $0^{\circ}$ to $180^{\circ}$, removing ambiguities in extrapolating data into angles inaccessible in earlier experiments.

The fitting procedure determined four parameters: the scattering length and the effective range $A$ and $R_{0}$ for $s$-waves and $B_{1}$ and $R_{1}$ for $p$-waves. The analysis is based on a detailed (performed with a $0.02 \mathrm{eV}$ step) series of experimental DCSs in the $0.11-2.0 \mathrm{eV}$ energy range for $45^{\circ}$, $90^{\circ}, 135^{\circ}$ and $180^{\circ}$ [31], see Figure 1 . However, due to the predicted limitation of the presently used MERT (the value of characteristic energy $E^{*}=0.76 \mathrm{eV}$ ), only DCSs below $1 \mathrm{eV}$ were used as input data for the fitting procedures. An unweighted least-square procedure simultaneously minimizing relative differences between MERT fit and all experimental points for all four angles was used. The results of the fit are shown in Figure 1, and the corresponding parameters of the effective range expansion are given in Table 2. 

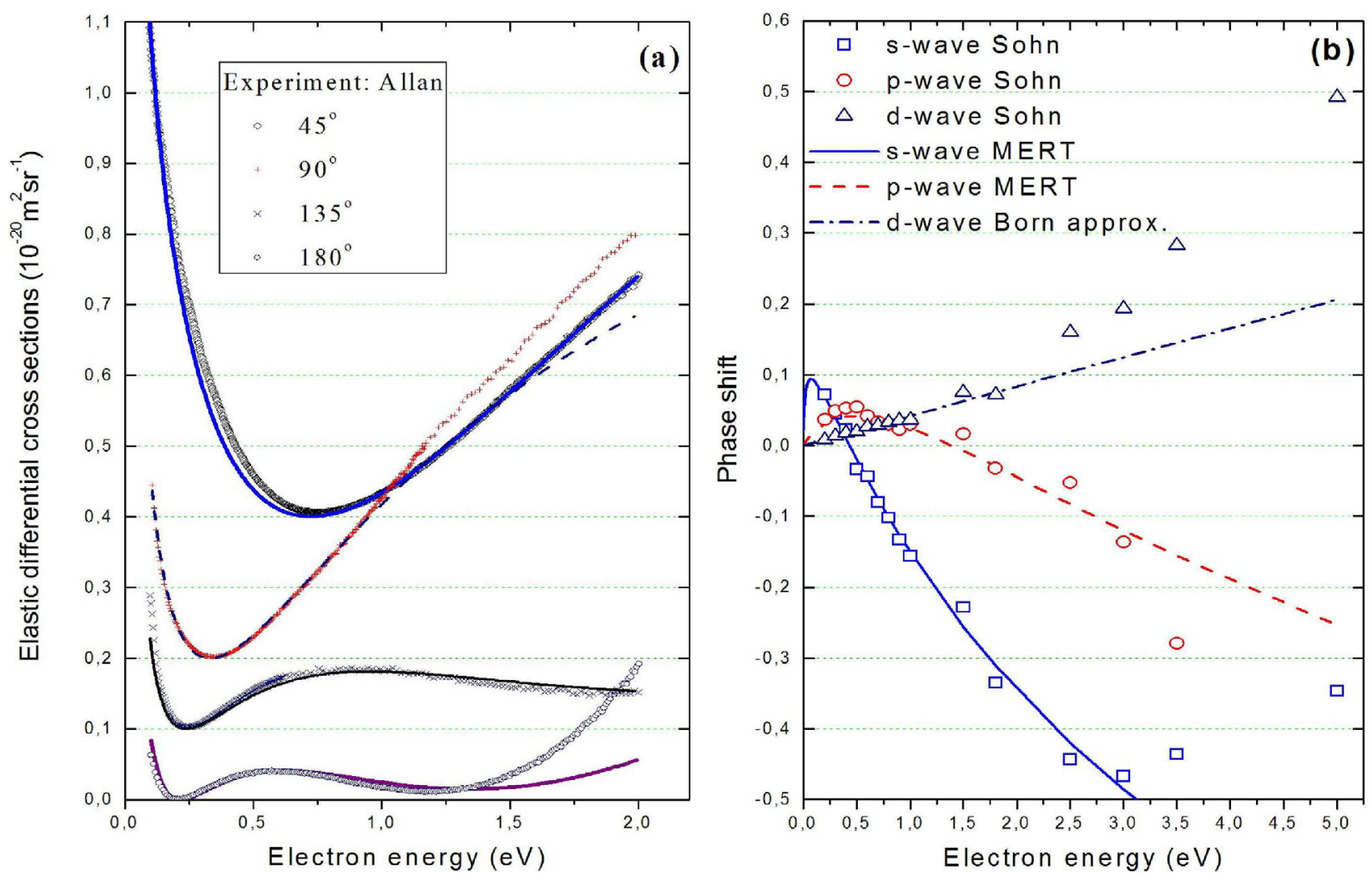

Fig. 1. (a) MERT simultaneous fit to four differential elastic cross-section datasets measured by Allan $[31]$ at $45^{\circ}, 90^{\circ}, 135^{\circ}$ and $180^{\circ}$. To improve visibility, the data for the three lowest angles are shifted by $0.4,0.2$ and $0.1 \AA^{2} /$ sr, respectively. (b) Present MERT-derived energy dependence of $s$ - and $p$-wave phase shifts (in radians) compared to the analysis of Sohn et al. [18]. The $d$-wave contribution calculated presently with Born approximation given by equation (4) is also shown for comparison.

The present MERT model practically coincides with Allan's experiment [31] within the error bar for $45^{\circ}$ in the range $0.2-2 \mathrm{eV}$, apart from slight discrepancies at $0.3-0.5 \mathrm{eV}$, where maxima for the vibrational excitations are located (compare with Fig. 3). At $90^{\circ}$ scattering angle a very good agreement is seen up to $1.1 \mathrm{eV}$, whilst for $135^{\circ}$ very good agreement can be seen in the whole range $0.2-2 \mathrm{eV}$. In the case of $180^{\circ}$ the theoretical curve remains within the experimental error bars (25\% [30]) up to $1.3 \mathrm{eV}$. In light of these results we conclude that the present MERT approach is able to reproduce with sufficient accuracy all four curves up to an impact energy of $1 \mathrm{eV}$, in agreement with the predicted characteristic energy.

To check the analysis, in Figure 1b we show the scattering phase-shifts for the three first partial waves derived using parameters from Table 2. Present results agree well with the semi-empirical analysis of Sohn et al. [18] even up to $2 \mathrm{eV}$, where a limit for the $d$-wave in the Born approximation, equation (4), is clearly posed. A small difference at low energies appears only at four points for the $p$-wave shift, at $0.2-0.5 \mathrm{eV}$, where the results of Sohn et al. [18] lie systematically at a constant value above our MERT curve. Such a difference (in the analysis, not measurements, see their Fig. 2 in Ref. [18]) overestimates, via extrapolation to scattering angles above $140^{\circ}$, the integral elastic cross section. The present calculations are not affected by this uncertainty because they are based on measurements at angles up to $180^{\circ}$.

Note, however, that the agreement between present and Sohn's analysis in Figure 1b is only indicative. A more precise analysis should involve considerations on the scattering geometry, see for example reference [45]. Note also that the present analysis does not derive the $d$-wave shift but uses the simple approximation, equation (4).

In Figure 2 we compare our MERT-derived differential CS versus scattering angle with available experimental results $[18,26,31,46]$ and some chosen theories [20,22-24]. Although DCSs vary quickly across the range of the R-T minimum, the presently used MERT analysis reproduces the shape of the DCS variations very well. As can be seen in Figure 2a MERT is in a good agreement with the experiment by Sohn et al. [18] at $0.2 \mathrm{eV}$, being lower by about $10 \%$ only in the $30-80^{\circ}$ angular range. Present MERT agrees also with the theoretical approach of Jain et al. [20] at this energy point.

At the absolute R-T minimum, $0.4 \mathrm{eV}$ in Figure 2c, the agreement between MERT analysis and the two experiments, Sohn et al. [18] and Allan [31], is somewhat poorer. In this case the discrepancy cannot be attributed to the experiments, but rather to some subtle elastic-inelastic channel coupling effects, probably due to the presence of vibrational excitation maxima. Note that the present MERT analysis coincides at this energy with one of the models 

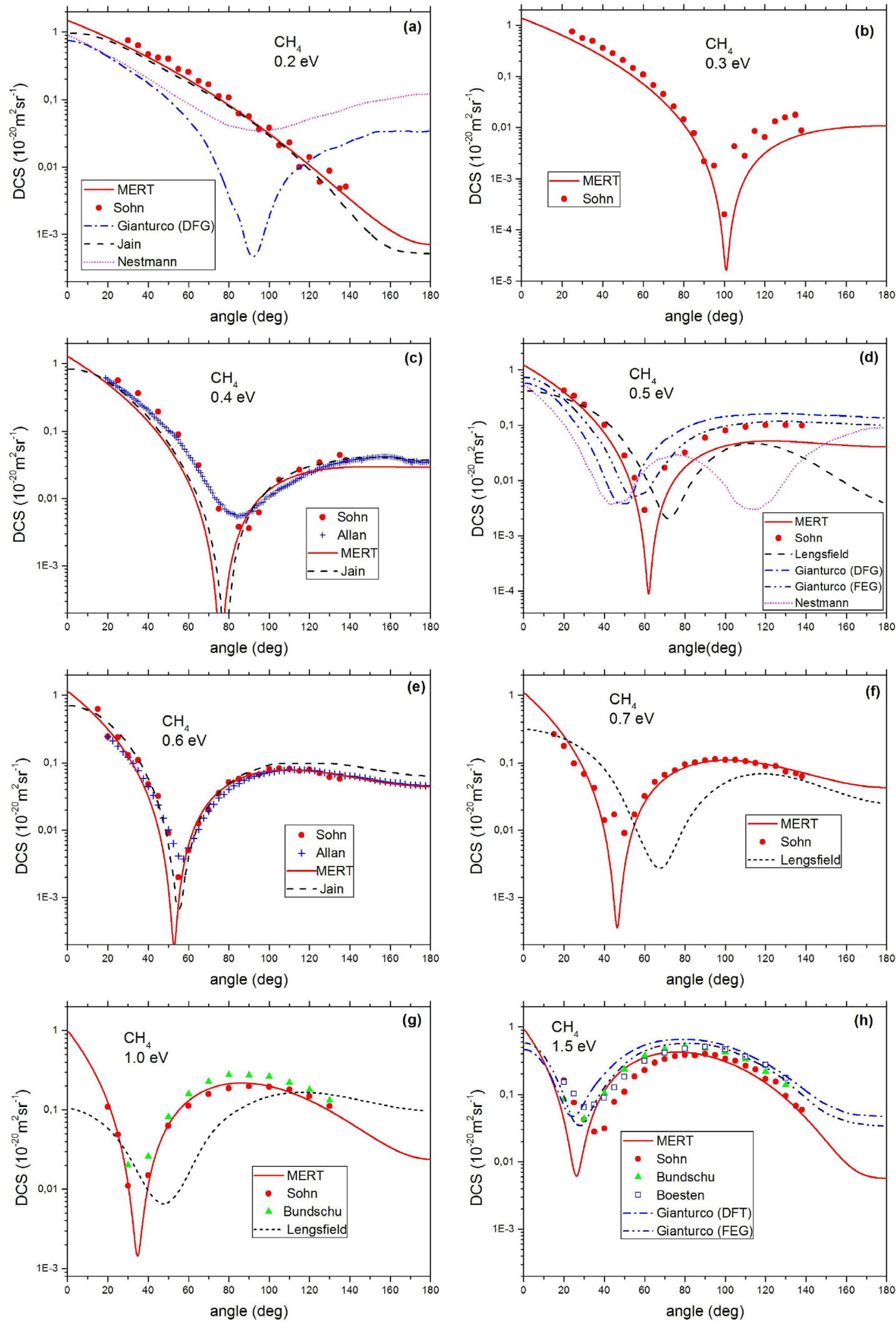

Fig. 2. MERT analysis (solid line) of differential elastic cross section versus the scattering angle at $0.2 \mathrm{eV}, 0.3 \mathrm{eV}, 0.4 \mathrm{eV}$, $0.5 \mathrm{eV}, 0.6 \mathrm{eV}, 0.7 \mathrm{eV}, 1 \mathrm{eV}$ and $1.5 \mathrm{eV}$, respectively. Experimental data are from Sohn et al. [18] (filled circles), Allan [31] (crosses), Bundschu et al. [26] (triangles) and Boesten and Tanaka [46] (open squares). Selected theoretical results are shown for comparison: density functional theory (DFT) and free-electron-gas model (FEG) by Gianturco et al. [24]; correlation-polarization model potential by Jain et al. [20], variational R-matrix by Nestmann et al. [23] and complex Kohn' method with polarized virtual orbitals by Lengsfield et al. [22]. 


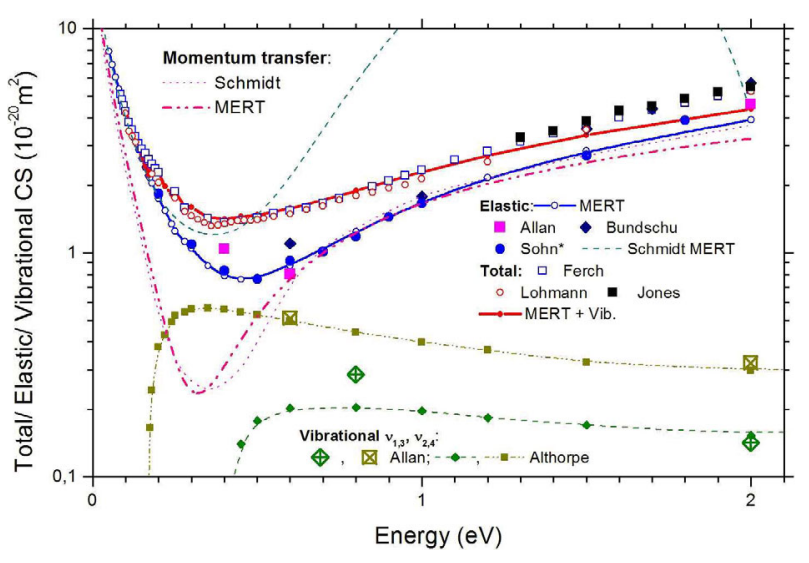

Fig. 3. Comparison of integral elastic, integral vibrational and total cross sections in methane. Elastic: present MERT - solid line with open circles; Bundschu et al. [26] - diamonds; Sohn et al. [18] - circles, at $0.2-0.5 \mathrm{eV}$ reduced arbitrarily by $0.5 \AA^{2}$, see text for discussion; six-parameter MERT analysis by Schmidt [29] - short dashed line. Vibrational CS for the two unresolved $\left(v_{1}+v_{3}\right)$ and $\left(v_{2}+v_{4}\right)$ modes: Althorpe et al. [45] - lines with diamonds and squares, respectively; Allan [30] $0-180^{\circ}$ DCS presently integrated - big diamonds and squares. Total: present elastic MERT plus two vibrational modes from Althorpe et al. [45] - line with solid circles; experimental data by Ferch et al. [35] - small open squares; Lohmann and Buckman [36] - small open circles. Momentum transfer: present MERT - dash-dot-dot line; swarm-derived by Schmidt [29] - dotted line.

by Jain et al. [20]. However, in the Jain's model [16,17] a phenomenological form of the polarization potential was used and the cut-off parameter was adjusted to reproduce the experimental R-T minimum. The present model is free of such adjustments; the position of the R-T minimum is defined intrinsically by the analysis of DCSs over a broad energy range.

At $0.5 \mathrm{eV}$ (Fig. 2d) MERT agrees with the experiment [18] much better than other theories do. At $0.6 \mathrm{eV}$ (Fig. 2e) MERT analysis reproduces perfectly the two available experiments, Sohn et al. [18] and Allan [31], above $60^{\circ}$ and below $40^{\circ}$; the somewhat deeper theoretical minimum than the experimental one could derive from the finite angular resolution of the measurements. An even better agreement between Sohn's experiment and the present analysis is found at $0.7 \mathrm{eV}$ (Fig. 2f). MERT works well also at $1.0 \mathrm{eV}$ and, surprisingly, even at $1.5 \mathrm{eV}$, see Figures $2 \mathrm{~g}$ and $2 \mathrm{~h}$. Present analysis starts to diverge at $2 \mathrm{eV}$ (not shown here), where it reproduces the shape of DCS variations but it underestimates the high-angle values. Obviously, the $d$-wave contribution should be treated more accurately at this energy, as is visible already in comparison with the experimental values in Figure 1a and in the phase-shift analysis, Figure 1b. It is difficult to find the exact $d$-wave phase-shift with current MERT analysis since the contribution of the $d$-wave to short-range interaction is very small in the considered energy range. Consequently, the parameters of the $d$-wave effective range expansion derived through the fitting procedure are nu- merically unstable. Hence the $d$-wave phase-shifts calculated in the Born approximation (Eq. (4)) have to be considered as sufficient to include this contribution.

\section{Integral cross sections}

Integral cross sections served as an additional check of the consistency of the MERT parameterization; for plasma modelling these cross sections are input data for deriving drift coefficients via the Boltzmann equation [38]. For this check we used experimental total and elastic cross sections as well as experimental and theoretical vibrational integral cross sections.

(1) Experimental total cross sections were measured from $0.085 \mathrm{eV}$ by Ferch et al. [35], from $0.1 \mathrm{~V}$ by Lohmann and Buckman [36] and from $1.3 \mathrm{eV}$ by Jones [37]; their data are shown in Figure 3. Measurements by Zecca et al. [47], coinciding within the error bar with those of Jones, are given only at a few energy points and with only two-digit precision, so they do not introduce any new information and they are not shown in Figure 3.

(2) Experimental integral elastic cross sections in our range of interest were given (using the closed-coupled model of Gianturco et al. [25]) in Bundschu et al. [26] at $0.6-2 \mathrm{eV}$ and by Sohn et al. [18] at $0.2-2 \mathrm{eV}$ (using a partial-wave fit). We note that the measurements of Sohn et al. [18] above and below $0.5 \mathrm{eV}$ come from two distinct experimental series, so some differences in normalization and/or analysis procedures are not to be excluded. Allan [31] more recently measured absolute DCS in the $19^{\circ}-179^{\circ}$ angular range and with $1^{\circ}$ step at $0.4,0.6$ and $2.0 \mathrm{eV}$. The error due to our numerical integration and extrapolation of his data is below $10 \%$. Bunschu et al.'s [26] integral and Allan's [31] integrated elastic cross sections are shown in Figure 3. Integral cross sections by Sohn et al. shown in Figure 3 at $0.2-0.5 \mathrm{eV}$ have been arbitrarily shifted in amplitude by $0.5 \times 10^{-20} \mathrm{~m}^{2}$ for the reasons discussed above.

(3a) Vibrational excitation data in the region of the R-T minimum obtained from the Kaiserslautern laboratory have been reported in several papers [18,48], but no integral cross sections were given. Curik et al. [15] compared their theoretical results to the recent experiment by Allan [30], but integral cross sections are given only above $2 \mathrm{eV}$. Allan [30] published separately energy dependences of vibrationalexcitation DCS at $90^{\circ}$ for $v_{1}, v_{2}, v_{3}$ and $v_{4}$ from thresholds up to $2 \mathrm{eV}$ modes what would allow a rough evaluation of the integral values, but the error on such a procedure is high (of order 50\%). Therefore, experimentally reliable data for deriving the integral vibrational-excitation cross sections remain exclusively those of Allan's [31] DSC measurements at $0.6 \mathrm{eV}$ and $2.0 \mathrm{eV}$ for the unresolved $v_{2}+v_{4}$ modes and at $0.8 \mathrm{eV}$ and $2.0 \mathrm{eV}$ for the $v_{1}+v_{3}$ mode. 
Our integration error on these data is not higher than 10\%. The integral values are shown in Figure 3.

(3b) Due to the lack of vibrational cross sections measured in beam experiments over a continuous energy scale some additional data are needed to complete the consistency checks.

The use of data derived from swarm experiments $[29,49,50]$ would not bring new information; furthermore, different sets disagree significantly in magnitude. Practically, in the $\mathrm{R}-\mathrm{T}$ minimum region the only theoretical data other than the Born approximation are several models by Gianturco et al. $[45,51,52]$. These models differ somewhat in amplitude of the new-to-threshold maxima. Nishimura and Gianturco [52] performed close-coupling calculations using a semi-classical exchange and by adjusting the calculated polarizability to experimental data. Their $v_{1,3}$ cross sections show a sharper peak (of $0.35 \times 10^{-20} \mathrm{~m}^{2}$ ) than off-shell results by Cascella et al. [51] $\left(0.2 \times 10^{-20} \mathrm{~m}^{2}\right)$, which, in turn, agree with the most recent calculation by Curik et al. [15], but only if the latter data are shifted by $1 \mathrm{eV}$. For the $v_{2,4}$ mode the difference is reversed the results of Cascella et al. [51] agree without adjustment but the data of Nishimura and Gianturco [52] are slightly lower $\left(0.55 \times 10^{-20} \mathrm{~m}^{2}\right.$ vs. $0.5 \times 10^{-20} \mathrm{~m}^{2}$, respectively). On the other hand, the earlier calculations with an exact exchange potential [45] reproduce much better the shape of the low-energy maximum in the integral vibrational cross section than the adiabatic-nuclear-vibration model by Cascella et al. does [51].

Because of all these differences in recent results, we chose a theory with an exact exchange and a short-range polarization modelled by free-electron-gas; the calculation corrects the adiabatic nuclear vibration approach via the Lippman-Schwinger equation [45]. This model correctly reproduces the thresholds of vibrational modes and predicts resonant peaks at about $8 \mathrm{eV}$ in agreement with the recent measurements [15]. Integral values for the $v_{1,3}$ and $v_{2,4}$ modes are compared with the experiment [15] in Figure 3. The agreement for $v_{2,4}$ is perfect both at $0.6 \mathrm{eV}$ and $2.0 \mathrm{eV}$ while the $v_{1,3}$ mode is similarly perfect at $2 \mathrm{eV}$ but poor (with a difference of $0.1 \times 10^{-20} \mathrm{~m}^{2}$ ) at $0.8 \mathrm{eV}$. Nevertheless, such a difference does not compromise the rest of the consistency check, affecting the summed cross section by not more than $10 \%$.

Resuming, as seen from Figure 3, the agreement of MERT analysis with the integral elastic CS of Sohn et al. [18] is very good in the whole energy range up to $1.5 \mathrm{eV}$ once their $0.2-0.5 \mathrm{eV}$ data are reduced by a constant value of $0.5 \AA^{2}$, as discussed in Figure 1b. Similarly, MERT agrees with the experiment by Bundschu et al. [26] within the declared experimental uncertainties (20-30\%). The present integral elastic MERT results summed with the integral vibrational cross sections of Althorpe et al. [45] agree with the three sets of total cross sections within less than the declared TCS measurement error bars. The difference observed in Figure 3 between experimental TCS and our summed (elastic + vibrational) values is within $10 \%$ in the whole $0.085-2.0 \mathrm{eV}$ energy range.
For comparison, in Figure 3 we show also the integral elastic cross section derived using Schmidt's six-parameter MERT approach, equation (3), with parameters given in Section 2 of this paper. Such a model works well only below $0.4 \mathrm{eV}$ but diverges completely with experiments at higher energies.

In Figure 3 we show additionally momentum transfer cross sections: the presently obtained momentum transfer CS resembles that proposed by Schmidt [29], i.e. derived directly from swarm experiments using multi-term analysis of the Boltzmann transport equation. Both curves are characterized by a similar deep minimum $\left(0.25 \AA^{2}\right)$, deeper by approximately a factor of two than those in some other swarm-derived cross-sections sets [27,49,50], the recent recommended data [53] or the latest, multiauthor collection of cross sections for $\mathrm{CH}_{4}$ from Boltzmann analysis, available on the Internet [54]. Note that for the relationship between the phase shifts, equations (1) and (2), the minimum in the momentum transfer CS should be deeper than that in the integral elastic CS, which excludes some of the CS sets [27] despite of their (apparent) agreement with swarm analysis.

The practical check of present momentum transfer CS was done via two-term Boltzmann analysis [38]. The calculated diffusion coefficients (drift velocity and transversal diffusion over the mobility ratio) agree with swarm experiments $[29,55-60]$ within $10 \%$ in the whole $0.01-10 \mathrm{Td}$ range of reduced-electric field, see Figure 4. However, it was shown already in the past that in the case of methane Boltzmann's multi-term $[61,62]$ or Monte Carlo [63] methods are needed. The first trials using multiterm Boltzmann analysis [64] show that the agreement is somewhat better than the two-term calculations presented in our Figure 4.

Note also, that Berkhan and Schmidt [65] postulated an increase of the rotational cross sections, just at the very $\mathrm{R}$-T minimum. They evaluated a maximum of the integral rotational cross section of $0.033 \times 10^{-20} \mathrm{~m}^{2}$ at $0.4 \mathrm{eV}$ what would perfectly "fill up" the difference between present MERT and experimental DCS seen in Figure 2. Unfortunately, none of the theories of rotational excitation that we are aware $[27,66,67]$ reported a maximum of the cross section at the R-T minimum. Further theoretical research would be important.

\section{Conclusions}

The present work aimed to summarize several experimental and theoretical works in order to cross-check the differential, elastic integral, integral vibrational, momentum transfer and total cross sections in methane. For this purpose we used an analytical form [32] of the modified effective range theory using only four parameters characterising the scattering potential. The analysis was based on recent [31] extensive measurements of DCS at four $\left(45^{\circ}\right.$, $90^{\circ}, 135^{\circ}$ and $180^{\circ}$ ) angles between 0.1 and $2.0 \mathrm{eV}$ of impact energy, thus removing ambiguities in extrapolating data into angles inaccessible in earlier experiments. 

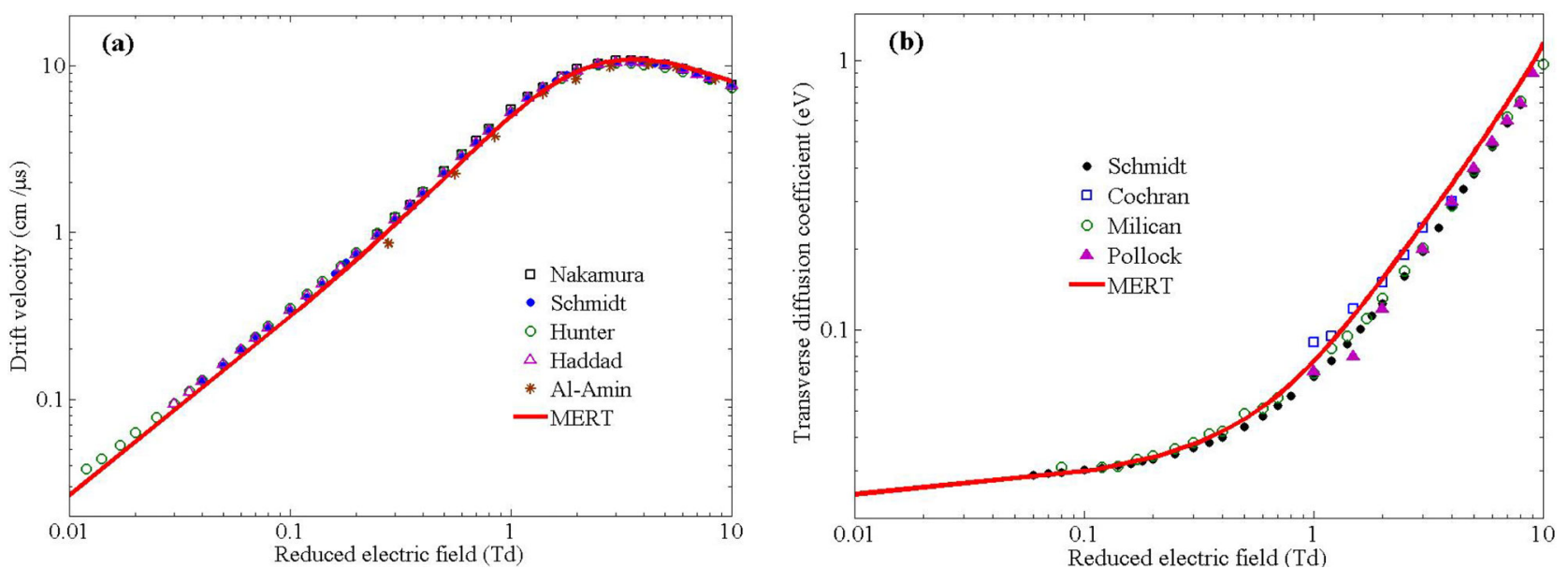

Fig. 4. Experimental swarm parameters: (a) the drift velocity and (b) the transverse diffusion coefficient, compared to solutions of two-term Boltzmann equation [38] using MERT-derived momentum transfer cross-section as the input data. The experimental results are from Schmidt [29], Hunter et al. [55], Haddad [56], Al-Amin et al. [57], Cochran and Forester [58], Millican and Walker [59] and Pollock [60].

Table 3. Recommended integral cross sections in the region of Ramsauer-Townsend minimum, used in present analysis. Abbreviations stand for elastic $\left(\sigma_{\mathrm{IE}}\right)$ presently obtained via MERT analysis, theoretical vibrational-excitation [45] for two unresolved experimentally modes $\left(\sigma_{2,4}+\sigma_{1,3}\right)$, summed values $\sigma_{\mathrm{sum}}=\sigma_{\mathrm{IE}}+\sigma_{2,4}+\sigma_{1,3}$. Scattering energies $(E)$ are given in eV; the cross sections are given in $\AA^{2}$.

\begin{tabular}{|c|c|c|c|c|c|c|c|c|c|c|c|c|c|c|}
\hline$E$ & $\sigma_{I E}$ & $\sigma_{2,4}$ & $\sigma_{1,3}$ & $\sigma_{\text {sum }}$ & $E$ & $\sigma_{I E}$ & $\sigma_{2,4}$ & $\sigma_{1,3}$ & $\sigma_{\text {sum }}$ & $E$ & $\sigma_{I E}$ & $\sigma_{2,4}$ & $\sigma_{1,3}$ & $\sigma_{\text {sum }}$ \\
\hline $10^{-3}$ & 24.11 & - & - & 24.11 & 0.175 & 2.290 & 0.178 & - & 2.486 & 0.450 & 0.759 & 0.543 & 0.145 & 1.447 \\
\hline $2.5 \times 10^{-3}$ & 22.11 & - & - & 22.11 & 0.200 & 1.890 & 0.381 & - & 2.271 & 0.475 & 0.761 & 0.536 & 0.163 & 1.459 \\
\hline $5 \times 10^{-3}$ & 19.95 & - & - & 19.95 & 0.225 & 1.586 & 0.456 & - & 2.046 & 0.500 & 0.772 & 0.528 & 0.178 & 1.476 \\
\hline $7.5 \times 10^{-3}$ & 18.37 & - & - & 18.37 & 0.250 & 1.355 & 0.521 & - & 1.871 & 0.525 & 0.790 & 0.520 & 0.186 & 1.496 \\
\hline 0.010 & 17.11 & - & - & 17.11 & 0.275 & 1.179 & 0.541 & - & 1.721 & 0.550 & 0.814 & 0.512 & 0.193 & 1.515 \\
\hline 0.025 & 12.33 & - & - & 12.33 & 0.300 & 1.045 & 0.559 & - & 1.599 & 0.575 & 0.843 & 0.504 & 0.198 & 1.542 \\
\hline 0.050 & 8.294 & - & - & 8.29 & 0.325 & 0.945 & 0.562 & - & 1.502 & 0.600 & 0.876 & 0.496 & 0.202 & 1.578 \\
\hline 0.075 & 6.020 & - & - & 6.02 & 0.350 & 0.872 & 0.565 & - & 1.435 & 0.700 & 1.042 & 0.469 & 0.208 & 1.744 \\
\hline 0.100 & 4.556 & - & - & 4.55 & 0.375 & 0.820 & 0.561 & - & 1.381 & 0.800 & 1.241 & 0.441 & 0.207 & 1.888 \\
\hline 0.125 & 3.547 & - & - & 3.55 & 0.400 & 0.786 & 0.557 & 0.065 & 1.412 & 0.900 & 1.460 & 0.420 & 0.202 & 2.082 \\
\hline \multirow[t]{3}{*}{0.150} & 2.823 & - & - & 2.82 & 0.425 & 0.767 & 0.550 & 0.117 & 1.437 & 1.000 & 1.689 & 0.399 & 0.197 & 2.286 \\
\hline & & & & & & & & & & 1.500 & $2.852^{*}$ & 0.323 & 0.17 & \\
\hline & & & & & & & & & & 2.000 & $3.918^{*}$ & 0.298 & 0.298 & \\
\hline
\end{tabular}

*MERT values at $1.5-2.0 \mathrm{eV}$ are underestimated, by some $5-15 \%$, due to underestimation of $d$-wave phase-shift.

To choose recommended vibrational integral CS we performed a thorough screening of theories [15,45,51,52], beam experiments [30,31] and swarm analysis [29,49,50]. We found that present MERT-derived integral elastic CS $\left(\sigma_{\mathrm{IE}}\right)$ combined with the theoretical vibrational CS $\left(\sigma_{2,4}+\sigma_{1,3}\right)$ by Althorpe et al. [45] reproduced almost perfectly up to $1 \mathrm{eV}$ the absolute total CS obtained in several alternative-type experiments and extended to low energies $[35-37,47]$. Such a combination not only provides a quantitative agreement, but also, most of all, it reconstructs the detailed shape of the experimental data.

At higher energies $(>1 \mathrm{eV})$ the present analysis starts to underestimate the measured total CS, indicating the limitations of MERT applicability in $\mathrm{CH}_{4}$. Note that the present MERT, using only four parameters of the effective range expansion, improves significantly the original version of MERT where six parameters were needed [29] to reproduce experimental data below $0.4 \mathrm{eV}$ and extend the validity of MERT analysis up to $2.0 \mathrm{eV}$.

Numerical values for integral cross sections recommended by the present analysis and the corresponding scattering phase-shifts are given in Tables 3 and 4 .

The elastic data derived in this work have to be considered as the first approach towards the recommended cross sections in methane in the R-T minimum. In particular, more efforts are needed to improve the coefficients describing the vibrational excitation. Calculations of the latter fail to reproduce the faint threshold structures seen in some experimental spectra [30]. Therefore, some minor corrections in the regions of the Ramsauer-Townsend minimum, $0.3-0.5 \mathrm{eV}$, are still not to be excluded. Tests by multi-term Boltzmann analysis 
Table 4. Recommended momentum transfer cross section $\left(\sigma_{\mathrm{MT}}\right)$ and corresponding the $s$-wave $\left(\eta_{0}\right)$ and the $p$-wave $\left(\eta_{1}\right)$ scattering phase-shifts in the region of Ramsauer-Townsend minimum. Scattering energies $(E)$ are given in eV; the cross sections are given in $\AA^{2}$ and the phase-shifts in radians.

\begin{tabular}{cccc|cccc|cccc}
\hline$E$ & $\sigma_{M T}$ & $\eta_{0}$ & $\eta_{1}$ & $E$ & $\sigma_{M T}$ & $\eta_{0}$ & $\eta_{1}$ & $E$ & $\sigma_{M T}$ & $\eta_{0}$ & $\eta_{1}$ \\
\hline 0.001 & 23.50 & 0.022 & 0.0003 & 0.175 & 0.87 & 0.075 & 0.0281 & 0.45 & 0.41 & -0.006 & 0.0406 \\
0.0025 & 21.20 & 0.034 & 0.0007 & 0.2 & 0.61 & 0.069 & 0.0304 & 0.475 & 0.46 & -0.014 & 0.0406 \\
0.005 & 18.76 & 0.046 & 0.001 & 0.225 & 0.44 & 0.061 & 0.0324 & 0.5 & 0.52 & -0.021 & 0.0405 \\
0.0075 & 16.99 & 0.054 & 0.002 & 0.25 & 0.33 & 0.054 & 0.0341 & 0.525 & 0.58 & -0.028 & 0.0403 \\
0.01 & 15.60 & 0.060 & 0.0026 & 0.275 & 0.27 & 0.047 & 0.0356 & 0.55 & 0.64 & -0.035 & 0.0399 \\
0.025 & 10.43 & 0.080 & 0.0061 & 0.3 & 0.24 & 0.039 & 0.0368 & 0.575 & 0.70 & -0.042 & 0.0395 \\
0.05 & 6.27 & 0.091 & 0.0111 & 0.325 & 0.23 & 0.031 & 0.0387 & 0.6 & 0.76 & -0.049 & 0.0367 \\
0.075 & 4.05 & 0.093 & 0.0155 & 0.35 & 0.25 & 0.024 & 0.0394 & 0.7 & 1.01 & -0.077 & 0.0333 \\
0.1 & 2.71 & 0.091 & 0.0193 & 0.375 & 0.27 & 0.016 & 0.0399 & 0.8 & 1.24 & -0.103 & 0.0289 \\
0.125 & 1.84 & 0.087 & 0.0226 & 0.4 & 0.31 & 0.009 & 0.0403 & 0.9 & 1.46 & -0.128 & 0.0237 \\
0.15 & 1.26 & 0.082 & 0.0256 & 0.425 & 0.36 & 0.001 & 0.0405 & 1.0 & 1.66 & -0.152 & 0.0181 \\
\hline
\end{tabular}

and Monte-Carlo models are also needed to obtain a consistent (i.e. better than 5\%) relationship between beam and swarm experimental results.

The high value of dipole polarizability $\left(\alpha \approx 19 a_{0}^{3}\right)$ that proved to work so well in the present analysis could indicate the importance of the octupole potential, which has the same dependence on distance as polarization. The value of the octupole moment is about $2-3 a_{0}^{3}$, compare [68]. The inclusion of all these effects is outside the scope of MERT and it requires more advanced approaches.

A possible next step towards analysing the R-T minima in other molecules, like $\mathrm{SiH}_{4}$ and $\mathrm{GeH}_{4}$ [4,49], using MERT would be the search for some systematic dependencies for the scattering lengths and the effective ranges. Without doubt $\mathrm{CH}_{4}$, with its high polarizability, proved to be a challenging target for MERT. Our estimate of the total uncertainty of the given cross sections is within $\pm 10 \%$ in the whole $0-1.5 \mathrm{eV}$ range. Different approaches are needed to perform similar semi-empirical analyses of elastic (and total) cross sections at higher energies.

We thank Prof. M. Allan for sending us extensive numerical sets of his experimental results and Dr Hab. Z. Idziaszek for providing us with detailed explanations of the MERT numerical package. The job was performed within Centre for Quantum Optics (COK UMK) and triggered by the International Atomic Energy Agency's interest in $\mathrm{CH}_{4}$ electron scattering. G.K. acknowledges the hospitality at the National Fusion Research Institute in Gunsan (Korea); K.F. would like to acknowledge the support of the Foundation for Polish Science.

\section{References}

1. C. Ramsauer, Ann. Phys. 66, 546 (1921)

2. J.S. Townsend, V.A. Bailey, Philos. Mag. 44, 1033 (1922)

3. J. Holstmark, Z. Phys. B 55, 437 (1929)

4. H. Cho, J.-S. Yoon, M.-Y. Song, Fusion Sci. Technol. 63, 349 (2013)

5. G.P. Karwasz, K. Fedus, Fusion Sci. Technol. 63, 338 (2013)

6. V.A. Godyak, V.I. Demidov, J. Phys. D 44, 233001 (2011)
7. C. Ramsauer, R. Kollath, Ann. Phys. 4, 91 (1930)

8. G.P. Karwasz, A. Zecca, R.S. Brusa, Riv. Nuovo Cim. 24(1), 1 (2001)

9. G.P. Karwasz, A. Zecca, R.S. Brusa, Riv. Nuovo Cim. 24(4), 1 (2001)

10. G. Ramanan, G.R. Freeman, J. Chem. Phys. 93, 3120 (1990)

11. Cz. Szmytkowski, K. Maciąg, G. Karwasz, D. Filipović, J. Phys. B 22, 525 (1989)

12. K. Jung, T. Antoni, R. Muller, K.-H. Kochem, H. Ehrhardt, J. Phys. B 15, 3535 (1982)

13. T.N. Olney, N.M. Cann, G. Cooper, C.E. Brion, Chem. Phys. 223, 59 (1997)

14. CRC Handbook of Chemistry and Physics, edited by D.R. Lide (CRC Press, Boca Raton, 1998)

15. R. Čurik, P. Cársky, M. Allan, J. Phys. B 41, 115203 (2008)

16. A. Jain, Phys. Rev. A 34, 3707 (1986)

17. A. Jain, D.G. Thomson, J. Phys. B 15, L631 (1982)

18. W. Sohn, K.H. Kochem, K.M. Scheuerlein, K. Jung, H. Ehrhardt, J. Phys. B 19, 3625 (1986)

19. F.A. Gianturco, A. Jain, L.C. Pantano, J. Phys. B 20, 571 (1986)

20. A. Jain, C.A. Weatherford, D.G. Thompson, P. McNaughten, Phys. Rev. A 40, 6730 (1989)

21. P. McNaughten, D.G. Thompson, A. Jain, J. Phys. B 23, 2405S (1990)

22. B.H. Lengsfield III, T.N. Rescigno, C.W. McCurdy, Phys. Rev. A 44, 4296 (1991)

23. B.M. Nestmann, K. Pfingst, S.D. Peyerimhoff, J. Phys. B 27, 2297 (1994)

24. F.A. Gianturco, J.A. Rodriguez-Ruiz, N. Sanna, J. Phys. B 28, 1287 (1995)

25. F.A. Gianturco, J.A. Rodriguez-Ruiz, N. Sanna, Phys. Rev. A 52, 1257 (1995)

26. C.T. Bundschu, J.C. Gibson, R.J. Gulley, M.J. Brunger, S.J. Buckman, N. Sanna, F.A. Gianturco, J. Phys. B 30, 2239 (1997)

27. H. Alvarez-Pol, I. Durany, R. Lorenzo, J. Phys. B 30, 2455 (1997)

28. H.N. Varambhia, J.J. Munro, J. Tennyson, Int. J. Mass Spectrom. 271, 1 (2008)

29. B. Schmidt, J. Phys. B 24, 4809 (1991)

30. M. Allan, J. Phys. B 38, 1679 (2005) and private information (2013) 
31. M. Allan, in 5th International Conference on Atomic and Molecular Data and Their Applications - AIP Conference Proceedings (2007), Vol. 901, p. 107

32. Z. Idziaszek, G.P. Karwasz, Phys. Rev. A 73, 064701 (2006)

33. Z. Idziaszek, G. Karwasz, Eur. Phys. J. D 51, 347 (2009)

34. K. Fedus, G.P. Karwasz, Z. Idziaszek, Phys. Rev. A 88, 012704 (2013)

35. J. Ferch, B. Granitza, W. Raith, J. Phys. B 18, L445 (1985)

36. B. Lohmann, S.J. Buckman, J. Phys. B 19, 2565 (1986)

37. R.K. Jones, J. Chem. Phys. 82, 5424 (1985)

38. G.J.M. Hagelaar, L.C. Pitchford, Plasma Sources Sci. Technol. 14, 722 (2005)

39. T.F. O’Malley, L. Spruch, L. Rosenberg, J. Math. Phys. 2, 491 (1961)

40. T.F. O'Malley, L. Rosenberg, L. Spruch, Phys. Rev. 125, $1300(1962)$

41. S.J. Buckman, J. Mitroy, J. Phys. B 22, 1365 (1989)

42. S.L. Lunt, J. Randell, J.P. Ziesel, G. Mrotzek, D. Field, J. Phys. B 27, 1407 (1994)

43. S.L. Lunt, J. Randell, J.P. Ziesel, G. Mrotzek, D. Field, J. Phys. B 31, 4225 (1998)

44. Z. Idziaszek, A. Simoni, T. Calarco, P.S. Julienne, New J. Phys. 13, 083005 (2011)

45. S.C. Althorpe, F.A. Gianturco, N. Sanna, J. Phys. B 20, 4165 (1995)

46. L. Boesten, H. Tanaka, J. Phys. B 24, 821 (1991)

47. A. Zecca, G. Karwasz, R.S. Brusa, C. Szmytkowski, J. Phys. B 24, 2747 (1991)

48. W. Sohn, K. Jung, H. Ehrhardt, J. Phys. B 16, 891 (1983)

49. D.K. Davies, L.E. Kline, W.E. Bies, J. Appl. Phys. 65, 3311 (1989)

50. M. Kurachi, Y. Nakamura, in Proc. 13th Symp. on ISIAT'90, Tokyo (1990), p. 205

51. M. Cascella, R. Curik, F.A. Gianturco, J. Phys. B 34, 705 (2001)

52. T. Nishimura, F.A. Gianturco, J. Phys. B 35, 2873 (2002)
53. M.T. Elford, S.J. Buckman, M. Brunger, in LandoltBörnstein - Numerical Data and Functional Relationships in Science and Technology, Photon and Electron Interactions with Atoms, Molecules and Ions (SpringerVerlag, Berlin, 2003), Vol. 17, p. 6085

54. S. Pancheshnyi, S. Biagi, M.C. Bordage, G.J.M. Hagelaar, A.V. Phelps, L.C. Pitchford, Chem. Phys. Lett. 398, 148 (2012)

55. S.R. Hunter, J.G. Carter, L.G. Christophorou, J. Appl. Phys. 60, 24 (1986)

56. G.N. Haddad, Aust. J. Phys. 38, 677 (1985)

57. S.A.J. Al-Amin, H.N. Kücükarpaci, J. Lucas, J. Phys. D 18, $1781(1985)$

58. L.W. Cochran, D.W. Forester, Phys. Rev. 126, 1785 (1962)

59. P.G. Millican, I.C. Walker, J. Phys. D 20, 193 (1987)

60. W.J. Pollock, Trans. Faraday Soc. 64, 2919 (1968)

61. K.F. Ness, R.E. Robson, Phys. Rev. 34, 2185 (1986)

62. B. Schmidt, S. Polenz, Nucl. Instrum. Methods A 273, 488 (1988)

63. Ž.D. Nikitović, A.I. Strinić, G.N. Malović, V.D. Stojanović, O.M. Šašic, Z.L. Petrović, Czech. J. Phys. Suppl. B 56, 958 (2006)

64. Y. Nakamura (from Keio University, Yokohama), private communication

65. K. Berkhan, B. Schmidt, private information (1984)

66. L.M. Brescansin, M.A.P. Lima, V. McKoy, Phys. Rev. A 40, 5577 (1989)

67. A. Jain, D.G. Thompson, J. Phys. B 16, 3077 (1983)

68. G. Birnbaum, E.R. Cohen, J. Chem. Phys. 62, 3807 (1975)

Open Access This is an open access article distributed under the terms of the Creative Commons Attribution License (http://creativecommons.org/licenses/by/4.0), which permits unrestricted use, distribution, and reproduction in any medium, provided the original work is properly cited. 